\title{
Koenigs-Knorr Synthesis of Cycloalkyl Glycosides
}

\author{
Zdeněk Wimmer $^{1, *}$, Lucie Pechová ${ }^{2}$ and David Šaman ${ }^{1}$ \\ ${ }^{1}$ Institute of Organic Chemistry and Biochemistry AS CR, Flemingovo náměstí 2, CZ-16610 \\ Prague 6, Czech Republic \\ ${ }^{2}$ Institute of Chemical Technology, Technická 5, CZ-16028 Prague 6, Czech Republic \\ * Author to whom correspondence should be addressed: E-mail: wimmer@uochb.cas.cz; \\ Tel.: (+420) 220183 281; FAX: (+420) 220183578
}

Received: 14 September 2004 / Accepted: 4 October 2004 / Published: 30 November 2004

\begin{abstract}
Cadmium carbonate was found to be a useful promoter in the Koenigs-Knorr synthesis of 2-(4-methoxybenzyl)cyclohexyl- $\beta$-D-glycopyranosides. Using this promoter model glucoside and galactoside derivatives of cyclic (i.e., secondary) alcohols were synthesized in 50-60\% overall yields. Diastereoisomeric mixtures of products were obtained in these syntheses, which started from racemic isomers of 2-(4-methoxybenzyl)cyclohexanol. The prepared compounds have been purified and characterized by their ${ }^{1} \mathrm{H}$ - and ${ }^{13} \mathrm{C}-\mathrm{NMR}$ spectra, as well as by their IR and MS spectra, in order to use them as reference compounds in planned subsequent research.
\end{abstract}

Keywords: Koenigs-Knorr synthesis; Cycloalkyl- $\beta$-D-glucopyranosides; Cycloalkyl- $\beta$ D-galactopyranosides; Cadmium carbonate.

\section{Introduction}

The Koenigs-Knorr method for the synthesis of glycosides and alkyl glycosides probably represents the most widely used procedure in the chemistry of carbohydrate derivatives. Application of the Koenigs-Knorr method confers a strong glycosyl donor character to the activated species by exchange of the anomeric hydroxyl functionality by a bromine or chlorine atom in the activation step [1-3]. The $\alpha$-halosubstituted carbohydrate generated in the activation step, can be readily further activated in the glycosylation step by halophilic promoters, i.e. heavy metal salts, resulting in an irreversible glycosyl transfer to the acceptor. This method is the basis of a valuable technique for the 
synthesis of complex alkyl glycosides, oligosaccharides or glycoconjugates. It has been continuously developed over the years and is widely employed [1-5], but in spite of the generality of the KoenigsKnorr method, its requirement for up to 4 equivalents of heavy metal salts as promoter can be a limiting factor for large-scale preparations.

More than 20 years ago, we had used cadmium carbonate [6] for a successful synthesis of 2-[4-(3ethoxy-3-methyl-1-butoxy)benzyl]cyclohexyl- $\beta$-D-glucopyranosides, derived from the separated racemic cis and trans isomers of 2-[4-(3-ethoxy-3-methyl-1-butoxy)benzyl]cyclohexanol, insect juvenile hormone bioanalogs [7]. At that time, only $100 \mathrm{MHz}$ NMR spectra of 2-[4-(3-ethoxy-3methyl-1-butoxy)benzyl]cyclohexyl- $\beta$-D-glucopyranoside products were measured, which ultimately were not published [6]. Recently, we have again focused our attention on alkyl glycosides. Although a remarkable variety of heavy metal salts have been reported to successfully promote the Koenigs-Knorr synthesis [2-5], we have applied our original method [6] again to thus obtain several reference compounds for a more comprehensive study of the glycosylation reaction. The currently used modification of the Koenigs-Knorr synthesis of alkyl glycosides has been proven to result in the formation of only the $\beta$-anomers of the alkyl glycoside products [2-6]. This selectivity is the key advantage of the method employed for the present study, undertaken with the purpose of obtaining detailed analytical data for the products. Using cadmium carbonate as the promoter of the KoenigsKnorr synthesis this time we obtained 2-(4-methoxybenzyl)cyclohexyl- $\beta$-D-glucopyranosides and - $\beta$-D-galactopyranosides. Our synthesis started from the separated racemic isomers of 2-(4methoxybenzyl)cyclohexanol, useful model compounds in the investigation of several reactions used for transformations of 2-substituted cycloalkanols into other desirable derivatives, and resulted in the obtention of diastereoisomeric mixtures of the respective products. From the ${ }^{1} \mathrm{H}$ - and ${ }^{13} \mathrm{C}$-NMR spectra of these products, as well as their IR and MS (FAB) spectra, valuable analytical data have been obtained. This study was also performed in order to develop and to prove the usefulness of a convenient method for our ongoing preparations of a series of biologically active alkyl glycosides, using the racemic isomers of 2-(4-methoxybenzyl)cyclohexanol as a model secondary alcohol.

\section{Results and Discussion}

The modification of the Koenigs-Knorr synthesis of alkyl glycosides used in the first synthetic step of the experiments described started from the separated racemic cis and trans isomers of 2-(4methoxybenzyl)cyclohexanol ( $\mathbf{1}$ and $\mathbf{2}$ ), and, therefore, the prepared products $\mathbf{3 a} / \mathbf{3 b}-\mathbf{6} \mathbf{a} / \mathbf{6} \mathbf{b}$, and subsequently also the products of the deprotection reaction $(\mathbf{7 a} / \mathbf{7 b}-\mathbf{1 0 a} / \mathbf{1 0 b})$, are diastereoisomeric mixtures (Scheme 1). A 1:1 ratio of the respective diastereoisomers was found in each mixture, which indicates that there was no detectable preference for either enantiomer of the isomeric racemic alcohols $\mathbf{1}$ and $\mathbf{2}$ during the Koenigs-Knorr synthesis. 


\section{Scheme 1}<smiles>COc1ccc(C[C@H]2CCCC[C@H]2O)cc1</smiles>

1, cis<smiles>COc1ccc(C[C@@H]2CCCC[C@H]2O)cc1</smiles>

2, trans

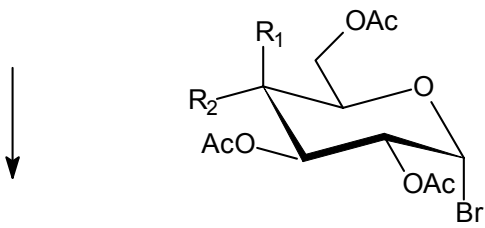<smiles></smiles>

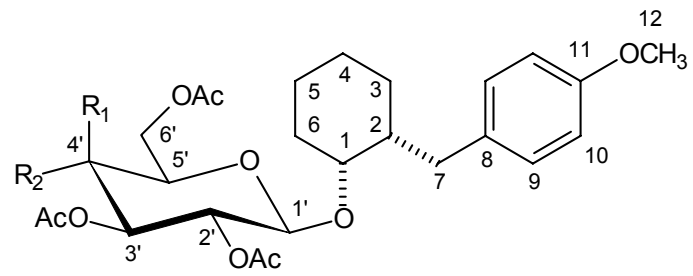

3a / 3b, cis $(\beta) ; \mathrm{R}_{1}=\mathrm{H}, \mathrm{R}_{2}=\mathrm{OAc}$ 4a / 4b, cis $(\beta) ; \mathrm{R}_{1}=\mathrm{OAc}, \mathrm{R}_{2}=\mathrm{H}$
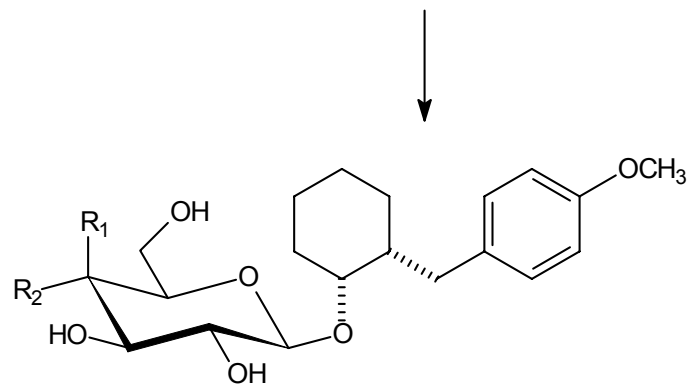

$7 \mathrm{a} / 7 \mathbf{b}, \operatorname{cis}(\beta) ; \mathrm{R}_{1}=\mathrm{H}, \mathrm{R}_{2}=\mathrm{OH}$ 8a / 8b, cis $(\beta) ; \mathrm{R}_{1}=\mathrm{OH}, \mathrm{R}_{2}=\mathrm{H}$

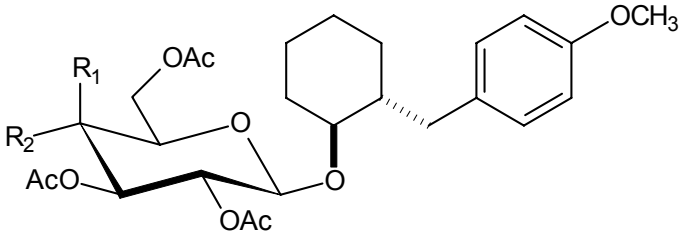

5a / 5b, trans $(\beta) ; \mathrm{R}_{1}=\mathrm{H}, \mathrm{R}_{2}=\mathrm{OAc}$ 6a / 6b, trans $(\beta) ; \mathrm{R}_{1}=\mathrm{OAc}, \mathrm{R}_{2}=\mathrm{H}$
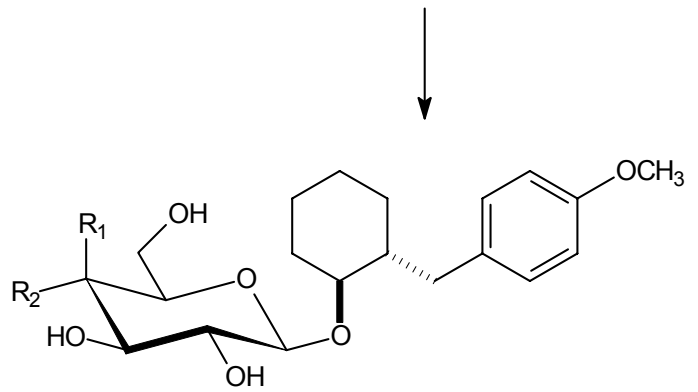

9a / 9b, trans $(\beta) ; \mathrm{R}_{1}=\mathrm{H}, \mathrm{R}_{2}=\mathrm{OH}$

10a / 10b, trans $(\beta) ; \mathrm{R}_{1}=\mathrm{OH}, \mathrm{R}_{2}=\mathrm{H}$

Our modification of the Koenigs-Knorr method consisted of maintaining rigorously anhydrous conditions for the whole duration of the reaction via continuous azeotropic removal of any humidity potentially present or entering the system, an approach that always resulted in the most advantageous reaction course for the Koenigs-Knorr synthesis of the alkyl glycoside derivatives $\mathbf{3 a} / \mathbf{3} \mathbf{b}-\mathbf{6 a} / \mathbf{6} \mathbf{b}$. The second synthetic step is a simple deprotection (removal of acetyl protecting groups from the carbohydrate moieties) of compounds $\mathbf{3 a} / \mathbf{3 b}-\mathbf{6 a} / \mathbf{6 b}$, using potassium carbonate in a 4:1 methanol / water medium [8] to give the final products $\mathbf{7 a} / \mathbf{7 b}-\mathbf{1 0 a} / \mathbf{1 0 b}$ in good yields (see Experimental).

It was found that the Koenigs-Knorr synthesis resulted in higher yields of the products $\mathbf{5 a} / \mathbf{5} \mathbf{b}$ and 6a/6b derived from the trans-alcohol 2 , a result which was expected due to higher steric hindrance during the substitution of the axial hydroxyl functionality in the cis-alcohol 1. In turn, deprotection of compounds $3 \mathbf{a} / \mathbf{3 b}-\mathbf{6 a} / \mathbf{6} \mathbf{b}$ took place faster and was more successful in the case of compounds $3 \mathbf{a} / \mathbf{3} \mathbf{b}$ and $\mathbf{4 a} / \mathbf{4 b}$, a finding that can be explained by the same steric hindrance, on account of the expected 
different molecular volumes of the respective glycosyloxy and 2',3',4',6'-tetra- $O$-acetylglycosyloxy groups, which also display different spatial orientations: thus, the $\mathrm{C}(1)-\mathrm{O}$ bond (Scheme 1) is axial in compounds $3 \mathbf{a} / \mathbf{3 b}, \mathbf{4 a / 4 b}, 7 \mathbf{a} / \mathbf{7 b}$ and $8 \mathbf{a} / \mathbf{8 b}$, and equatorial in compounds $5 \mathbf{a} / \mathbf{5 b}, \mathbf{6 a} / \mathbf{6 b}, 9 \mathbf{a} / \mathbf{9 b}$ and $\mathbf{1 0 a} / \mathbf{1 0 b}[9]$.

The synthesized diastereoisomeric mixtures of products $\mathbf{3 a} / \mathbf{3 b}-\mathbf{1 0 a} / \mathbf{1 0 b}$ were used as reference compounds for recording the desired model ${ }^{1} \mathrm{H}$ - and ${ }^{13} \mathrm{C}-\mathrm{NMR}$ spectra, supported by standard recording of their MS (FAB) and IR spectra. It is a great advantage of the applied modification of the Koenigs-Knorr synthesis [2] that only $\beta$-anomers of the products occur in the reaction mixtures. The ${ }^{1} \mathrm{H}-{ }^{1} \mathrm{H}$ PFG COSY and the ${ }^{1} \mathrm{H}_{-}{ }^{13} \mathrm{C}$ PFG HSQC experiments $[10,11]$ were performed using standard pulse programs, as provided by the spectrometer manufacturer, to unambiguously assign the signals in the recorded spectra. Characteristic parameters of the experiments are given in the Experimental part. The obtained data are presented in the Experimental part $\left({ }^{1} \mathrm{H}-\mathrm{NMR}\right.$ data) and in Tables 1 and 2 $\left({ }^{13} \mathrm{C}-\mathrm{NMR}\right.$ data).

\section{Conclusions}

The used modification of the Koenigs-Knorr synthesis [1-3] of alkyl glycosides has proven to be a valuable and convenient procedure for preparation of pure 2-(4-methoxybenzyl)cyclohexyl- $\beta$-Dglucopyranosides $(\mathbf{3 a} / \mathbf{3 b}$ and $\mathbf{5 a} / \mathbf{5 b})$ and $-\beta$-D-galactopyranosides $(\mathbf{4} \mathbf{a} / \mathbf{4} \mathbf{b}$ and $\mathbf{6 a} / \mathbf{6 b})$, i.e. glycosides derived from the secondary cyclic alcohols. It has also been proven by analysis of all minor compounds in the reaction mixtures resulting from the synthesis of the compounds $3 \mathbf{a} / \mathbf{3 b}-\mathbf{6 a} / \mathbf{6} \mathbf{b}$ that no $\alpha$-anomers of $3 \mathbf{a} / \mathbf{3 b}-\mathbf{6 a} / \mathbf{6} \mathbf{b}$ were formed during the reaction. Finally, it has been proven that this synthetic approach is a convenient method to be applied in the ongoing synthesis of biologically active alkyl- $\beta$-D-glucopyranosides and alkyl- $\beta$-D-galactopyranosides.

\section{Acknowledgments}

Financial support through the grant 203/02/0166 (Grant Agency of the Czech Republic) is gratefully acknowledged. The authors thank Mrs. M. Wimmerová for her skillful technical assistance.

\section{Experimental}

\section{General}

The ${ }^{1} \mathrm{H}-\mathrm{NMR}$ and the ${ }^{13} \mathrm{C}-\mathrm{NMR}$ spectra were recorded on a Bruker AVANCE 500 spectrometer (in FT mode) at $500.1 \mathrm{MHz}$ and $125.8 \mathrm{MHz}$, respectively, in $\mathrm{CDCl}_{3}$ or in $\mathrm{CD}_{3} \mathrm{OD}$, using either tetramethylsilane $\left(\delta 0.0-\mathrm{CDCl}_{3}\right)$ or a solvent signal $\left(\mathrm{CD}_{3} \mathrm{OD}, \delta 3.31\right.$ for ${ }^{1} \mathrm{H}$ - and $\delta 49.50$ for ${ }^{13} \mathrm{C}$ - $)$ as internal references. 2D-NMR experiments were measured using the following characteristic parameters: ${ }^{1} \mathrm{H}-{ }^{1} \mathrm{H}$ PFG COSY - spectral width 9 ppm in both $f_{1}, f_{2}$ dimensions, delay $1 \mathrm{~s}$, data matrix for processing $2048 \times 2048$ data points; ${ }^{1} \mathrm{H}_{-}{ }^{13} \mathrm{C}$ PFG HSQC - spectral width $9 \mathrm{ppm}$ in $f_{2}$ and $160 \mathrm{ppm}$ in $f_{l}$, delay $1 \mathrm{~s}$, data matrix for processing $2048 \times 2048$ data points. IR spectra were recorded in solution $\left(\mathrm{CCl}_{4}\right)$ or by the $\mathrm{KBr}$ technique on a Bruker IFS 88 instrument. MS (FAB) were recorded on a 
VG analytical 70 - 250 SE mass spectrometer, ZAB-EQ (BEQQ configuration) at $70 \mathrm{eV}$. Preparative column chromatography was performed on a silica gel type 60 (particle size $0.04-0.063 \mathrm{~mm}$, Fluka, Switzerland). TLC was performed on aluminum sheets precoated with silica gel 60 (Merck, Germany). Analytical HPLC was carried out on a TSP (Thermoseparation Products, USA) instrument equipped with a ConstaMetric 4100 Bio pump and a SpectroMonitor 5000 UV DAD. Product analyses were performed on a Sepharon SGX C-18 reverse phase column $(250 \times 4 \mathrm{~mm}$, particle size $7 \mathrm{~mm}$, Watrex, Czech Republic) or on a chiral Nucleodex $\beta-\mathrm{OH}$ column (150 x 4 mm, Macherey-Nagel, Germany) using 4:1 (v/v) methanol-water as mobile phase at $0.5 \mathrm{~mL} \mathrm{~min}^{-1}$ or at $0.3 \mathrm{~mL} \mathrm{~min}^{-1}$. The eluate was monitored at 220, 254 and $275 \mathrm{~nm}$ and UV spectra were run from 200 to $300 \mathrm{~nm}$.

2-(4-Methoxybenzyl)cyclohexyl-2,,3',4',6'-tetra-O-acetyl- $\beta$-D-glucopyranoside (3a/3b and $\mathbf{5 a} / \mathbf{5 b})$ and 2-(4-methoxybenzyl)cyclohexyl-2',3',4',6'-tetra-O-acetyl- $\beta$-D-galactopyranoside (4a/4b and 6a/6b).

The respective isomers of 2-(4-methoxybenzyl)cyclohexanol (0.272 g; $1.24 \mathrm{mmol})$ were dissolved in toluene $(30 \mathrm{~mL})$, and cadmium carbonate $(0.656 \mathrm{~g} ; 3.72 \mathrm{mmol})$ was added. A portion of the toluene ( 7 to $10 \mathrm{~mL}$ ) was distilled off under azeotropic conditions to remove all traces of water from the system. A solution of 2,3,4,6-tetra- $O$-acetyl- $\alpha$-D-glucopyranosyl bromide or 2,3,4,6-tetra- $O$-acetyl- $\alpha$ D-galactopyranosyl bromide $(1.528 \mathrm{~g} ; 3.72 \mathrm{mmol})$ in toluene $(2 \mathrm{~mL})$ was then added over 5 minutes to the reaction mixture, which was further stirred and heated under azeotropic conditions for an additional $6 \mathrm{~h}$. A mixture of inorganic salts was removed by filtration, and the solvent was evaporated from the filtrate, affording a dry residue. Silica gel column chromatography (eluent: $3: 1$ to $1: 1$ stepwise gradient of light petroleum - ether) yielded the pure products in yields of $61.6 \%(\mathbf{3 a} / \mathbf{3 b}), 61.8 \%$ $(\mathbf{4 a} / \mathbf{4 b}), 83.6 \%(\mathbf{5 a} / \mathbf{5 b})$ and $73.7 \%(\mathbf{6 a} / \mathbf{6 b})$, respectively.

3a/3b: ${ }^{1} \mathrm{H}-\mathrm{NMR}\left(\mathrm{CDCl}_{3}\right)$ : Diastereoisomer A: 1.17 - 1.43 (m, 6H, H-3a, 3b, 4a, 5a, 5b and 6a), 1.57 1.68 (m, 2H, H-2 and 4b), 1.84 (m, 1H, H-6b), 2.05 (s, 3H, OAc), 2.06 (s, 3H, OAc), 2.08 (s, 6H, $2 \times$ OAc), 2.41 (dd, $J=6.4,13.8 \mathrm{~Hz}, 1 \mathrm{H}, \mathrm{H}-7 \mathrm{a}), 2.72(\mathrm{dd}, J=7.8,13.8 \mathrm{~Hz}, 1 \mathrm{H}, \mathrm{H}-7 \mathrm{~b}), 3.65$ (ddd, $J=2.6$, 4.7, $10.0 \mathrm{~Hz}, 1 \mathrm{H}, \mathrm{H}-5$ '), 3.80 (s, 3H, H-12), 3.81 (dt, $J=2.4,2.4,4.3 \mathrm{~Hz}, 1 \mathrm{H}, \mathrm{H}-1$ ), 4.14 (dd, $J=2.6$, $12.2 \mathrm{~Hz}, 1 \mathrm{H}, \mathrm{H}-6$ 'a), 4.28 (dd, $J=4.7,12.2 \mathrm{~Hz}, 1 \mathrm{H}, \mathrm{H}-6$ 'b), 4.56 (d, $J=7.9 \mathrm{~Hz}, 1 \mathrm{H}, \mathrm{H}-1$ '), 5.11 (dd, $J=$ 7.9, 9.9Hz, 1H, H-2'), 5.15 (t, $J=9.8 \mathrm{~Hz}, 1 \mathrm{H}, \mathrm{H}-4$ '), 5.22 (t, $J=9.8 \mathrm{~Hz}, 1 \mathrm{H}, \mathrm{H}-3$ '), 6.77 (m, 2H, H-9), 7.13 (m, 2H, H-8); Diastereoisomer B: $1.17-1.43$ (m, 6H, H-3a, 3b, 4a, 5a, 5b and 6a), $1.57-1.68$ (m, 2H, H-2 and 4b), 1.84 (m, 1H, H-6b), 2.02 (s, 6H, $2 \times \mathrm{OAc}), 2.03$ (s, 6H, $2 \times \mathrm{OAc}), 2.39$ (dd, $J=$ $7.7,13.6 \mathrm{~Hz}, 1 \mathrm{H}, \mathrm{H}-7 \mathrm{a}), 2.53$ (dd, $J=7.0,13.6 \mathrm{~Hz}, 1 \mathrm{H}, \mathrm{H}-7 \mathrm{~b}), 3.64$ (ddd, $J=2.6,5.5,10.1 \mathrm{~Hz}, 1 \mathrm{H}, \mathrm{H}-$ 5'), 3.65 (dt, $J=2.4,2.4,4.5 \mathrm{~Hz}, 1 \mathrm{H}, \mathrm{H}-1$ ), 3.77 (s, 3H, H-12), 4.11 (dd, $J=2.6,12.2 \mathrm{~Hz}, 1 \mathrm{H}, \mathrm{H}-6$ 'a), $4.21\left(\mathrm{dd}, J=5.5,12.2 \mathrm{~Hz}, 1 \mathrm{H}, \mathrm{H}-6{ }^{\prime} \mathrm{b}\right), 4.55$ (d, $\left.J=7.9 \mathrm{~Hz}, 1 \mathrm{H}, \mathrm{H}-1^{\prime}\right), 5.06$ (dd, $J=9.3,10.1 \mathrm{~Hz}, 1 \mathrm{H}, \mathrm{H}-$ 4'), 5.09 (dd, $J=7.9,9.8 \mathrm{~Hz}, 1 \mathrm{H}, \mathrm{H}-2$ '), 5.23 (t, $J=9.5 \mathrm{~Hz}, 1 \mathrm{H}, \mathrm{H}-3$ '), 6.84 (m, 2H, H-10), 7.06 (m, 2H, H-9). IR ( $\left.\mathrm{cm}^{-1}, \mathrm{CCl}_{4}\right): 3027$ (w), 2960 (w), 2936 (w), 2836 (w), 1756 (s), 1613 (w), 1513 (m), 1441 (w), 1388 (m), 1300 (w), 1245 (s), 1220 (s), 1042 (s), 895 (w), 843 (w). MS (FAB): [M] 550 (4), 331 (28), 289 (5), 229 (6), 203 (24), 169 (56), 121 (100), 109 (40), $77(8)$. [ $\alpha]_{\mathrm{D}}{ }^{20}=-99$ (c 0.22 , $\mathrm{CHCl}_{3}$ ). For $\mathrm{C}_{28} \mathrm{H}_{38} \mathrm{O}_{11}$ (550.61): C 61.08, H 6.96; found: C 60.92, H 6.95. 
4a/4b: ${ }^{1} \mathrm{H}-\mathrm{NMR}\left(\mathrm{CDCl}_{3}\right)$ : Diastereoisomer A: 0.89 (m, 1H, H-3a), $1.18(\mathrm{~m}, 1 \mathrm{H}, \mathrm{H}-5 \mathrm{a}), 1.22(\mathrm{~m}, 1 \mathrm{H}$, H-4a), 1.36 (m, 1H, H-6), 1.57 m, 1H, H-5b), 1.62 (m, 1H, H-2), 1.63 (m, 1H, H-3b), 1.67 (m, 1H, H4b), 1.84 (m, 1H, H-6b), 2.00 (s, 3H, OAc), 2.02 (s, 3H, OAc), 2.09 (s, 3H, OAc), 2.17 (s, 3H, OAc), 2.44 (dd, $J=7.0,13.8 \mathrm{~Hz}, 1 \mathrm{H}, \mathrm{H}-7 \mathrm{a}), 2.74$ (dd, $J=7.4,13.8 \mathrm{~Hz}, 1 \mathrm{H}, \mathrm{H}-7 \mathrm{~b}), 3.80$ (s, 3H, H-12), 3.81 (dt, $J=2.3,2.3,4.5 \mathrm{~Hz}, 1 \mathrm{H}, \mathrm{H}-1), 3.87$ (dt, $J=1.2,6.7,6.7 \mathrm{~Hz}, 1 \mathrm{H}, \mathrm{H}-5$ '), $4.16(\mathrm{~d}, J=6.7 \mathrm{~Hz}, 2 \mathrm{H}, \mathrm{H}-$ 6a, H-6b), 4.51 (d, $\left.J=7.9 \mathrm{~Hz}, 1 \mathrm{H}, \mathrm{H}-1^{\prime}\right), 5.05$ (dd, $\left.J=3.5,10.5 \mathrm{~Hz}, 1 \mathrm{H}, \mathrm{H}-3^{\prime}\right), 5.30$ (dd, $J=7.9$, $10.5 \mathrm{~Hz}, 1 \mathrm{H}, \mathrm{H}-2$ '), 5.40 (dd, $J=1.2,3.5 \mathrm{~Hz}, 1 \mathrm{H}, \mathrm{H}-4$ '), 6.80 (m, 2H, H-10), 7.13 (m, 2H, H-9); Diastereoisomer B: 0.89 (m, 1H, H-3a), 1.18 (m, 1H, H-5a), 1.22 (m, 1H, H-4a), 1.36 (m, 1H, H-6), $1.57 \mathrm{~m}, 1 \mathrm{H}, \mathrm{H}-5 \mathrm{~b}), 1.62$ (m, 1H, H-2), 1.63 (m, 1H, H-3b), 1.67 (m, 1H, H-4b), 1.84 (m, 1H, H-6b), 2.00 (s, 3H, OAc), 2.02 (s, 3H, OAc), 2.06 (s, 3H, OAc), 2.09 (s, 3H, OAc), 2.41 (dd, J=8.0, 13.6Hz, $1 \mathrm{H}, \mathrm{H}-7 \mathrm{a}), 2.54$ (dd, $J=6.8,13.6 \mathrm{~Hz}, 1 \mathrm{H}, \mathrm{H}-7 \mathrm{~b}$ ), 3.66 (dt, $J=2.6,2.6,4.6 \mathrm{~Hz}, 1 \mathrm{H}, \mathrm{H}-1), 3.78$ (s, 3H, H12), 3.84 (dt, $J=1.2,6.7,6.7 \mathrm{~Hz}, 1 \mathrm{H}, \mathrm{H}-5$ '), 4.08 (dd, $J=6.8,11.2 \mathrm{~Hz}, 1 \mathrm{H}, \mathrm{H}-6$ 'a), 4.16 (dd, $J=6.6$, $11.2 \mathrm{~Hz}, 1 \mathrm{H}, \mathrm{H}-6$ 'b), 4.50 (d, $J=7.9,1 \mathrm{H}, \mathrm{H}-1^{\prime}$ ), 5.04 (dd, $J=3.5,10.5 \mathrm{~Hz}, 1 \mathrm{H}, \mathrm{H}-3$ '), 5.26 (dd, $J=7.9$, $\left.10.5 \mathrm{~Hz}, 1 \mathrm{H}, \mathrm{H}-2^{\prime}\right), 5.38$ (dd, $\left.J=1.2,3.5 \mathrm{~Hz}, 1 \mathrm{H}, \mathrm{H}-4^{\prime}\right), 6.84$ (m, 2H, H-10), 7.02 (m, 2H, H-9). IR (cm 1, $\left.\mathrm{CCl}_{4}\right): 3063$ (w), 3030 (w), 2936 (m), 2859 (w), 2836 (w), 1757 (s), 1613 (w), 1513 (s), 1441 (w), 1369 (s), 1300 (w), 1246 (s), 1221 (s), 1173 (s), 1056 (s), 1045 (s), 898 (w), 842 (w). MS (FAB): [M] $550(1), 331(10), 203(12), 169(24), 121(100), 109(28), 91(10)$. $[\alpha]_{\mathrm{D}}{ }^{20}=-70$ (c 0.24, $\left.\mathrm{CHCl}_{3}\right)$. For $\mathrm{C}_{28} \mathrm{H}_{38} \mathrm{O}_{11}$ (550.61): C 61.08, H 6.96; found: C 61.00, H 6.92.

5a/5b: ${ }^{1} \mathrm{H}-\mathrm{NMR}\left(\mathrm{CDCl}_{3}\right)$ : Diastereoisomer A: 0.88 (m, 1H, H-3a), 1.10 (m, 1H, H-5a), $1.22(\mathrm{~m}, 1 \mathrm{H}$, H-4a), 1.42 (m, 1H, H-6a), 1.56 (m, 1H, H-5b), 1.58 (m, 1H, H-2), 1.63 (m, 1H, H-3b), 1.72 (m, 1H, H-4b), 2.00 (s, 3H, OAc), 2.03 (s, 3H, OAc), 2.05 (s, 3H, OAc), 2.08 (s, 3H, OAc), 2.16 (m, 1H, H$6 \mathrm{~b}), 2.19$ (dd, $J=9.3,13.6 \mathrm{~Hz}, 1 \mathrm{H}, \mathrm{H}-7 \mathrm{a}), 3.13(\mathrm{dd}, J=3.6,13.6 \mathrm{~Hz}, 1 \mathrm{H}, \mathrm{H}-7 \mathrm{~b}), 3.32$ (dt, $J=4.2,9.5$, $9.5 \mathrm{~Hz}, 1 \mathrm{H}, \mathrm{H}-1), 3.71$ (ddd, $J=2.6,5.4,10.1 \mathrm{~Hz}, 1 \mathrm{H}, \mathrm{H}-5$ '), 3.78 (s, 3H, H-12), 4.17 (dd, $J=2.6$, $12.1 \mathrm{~Hz}, 1 \mathrm{H}, \mathrm{H}-6$ 'a), 4.27 (dd, $J=5.4,12.1 \mathrm{~Hz}, 1 \mathrm{H}, \mathrm{H}-6$ 'b), 4.63 (d, $J=8.0,1 \mathrm{H}, \mathrm{H}-1$ '), 5.08 (dd, $J=$ $\left.8.0,9.6 \mathrm{~Hz}, 1 \mathrm{H}, \mathrm{H}-2^{\prime}\right), 5.11$ (t, $J=9.8 \mathrm{~Hz}, 1 \mathrm{H}, \mathrm{H}-4$ '), 5.22 (t, $J=9.6 \mathrm{~Hz}, 1 \mathrm{H}, \mathrm{H}-3$ '), 6.81 (m, 2H), 7.09 $(\mathrm{m}, 2 \mathrm{H})$; Diastereoisomer B: $0.88(\mathrm{~m}, 1 \mathrm{H}, \mathrm{H}-3 \mathrm{a}), 1.10(\mathrm{~m}, 1 \mathrm{H}, \mathrm{H}-5 \mathrm{a}), 1.22(\mathrm{~m}, 1 \mathrm{H}, \mathrm{H}-4 \mathrm{a}), 1.42(\mathrm{~m}$, 1H, H-6a), 1.56 (m, 1H, H-5b), 1.58 (m, 1H, H-2), 1.63 (m, 1H, H-3b), 1.72 (m, 1H, H-4b), 2.00 (s, $3 \mathrm{H}, \mathrm{OAc}$ ), 2.03 (s, 3H, OAc), 2.05 (s, 3H, OAc), 2.08 (s, 3H, OAc), 2.09 (dd, $J=8.9,13.2 \mathrm{~Hz}, 1 \mathrm{H}, \mathrm{H}-$ 7a), 3.07 (dd, $J=3.3,13.2 \mathrm{~Hz}, 1 \mathrm{H}, \mathrm{H}-7 \mathrm{~b}$ ), 3.21 (dt, $J=4.3,9.7,9.7 \mathrm{~Hz}, 1 \mathrm{H}, \mathrm{H}-1$ ), 3.68 (ddd, $J=2.7$, 4.7, 10.0Hz, 1H, H-5'), 3.78 (s, 3H, H-12), 4.13 (dd, $J=2.7,12.1 \mathrm{~Hz}, 1 \mathrm{H}, \mathrm{H}-6$ 'a), 4.25 (dd, $J=4.7$, $12.1 \mathrm{~Hz}, 1 \mathrm{H}, \mathrm{H}-6$ 'b), 4.62 (d, $\left.J=8.0,1 \mathrm{H}, \mathrm{H}-1^{\prime}\right), 5.01$ (dd, $J=8.0,9.8 \mathrm{~Hz}, 1 \mathrm{H}, \mathrm{H}-2$ '), 5.10 (t, $J=9.8 \mathrm{~Hz}$, $1 \mathrm{H}, \mathrm{H}-4$ '), 5.22 (t, $J=9.6 \mathrm{~Hz}, 1 \mathrm{H}, \mathrm{H}-3$ '), 6.80 (m, 2H, H-10), 7.02 (m, 2H, H-9). IR ( $\left.\mathrm{cm}^{-1}, \mathrm{CCl}_{4}\right): 3031$ (w), 2935 (m), 2859 (w), 2853 (w), 1761 (s), 1753 (s), 1613 (w), 1513 (m), 1442 (w), 1300 (w), 1246 (s), 1225 (s), 1040 (s), $880(\mathrm{w}), 856$ (w). MS (FAB): [M] 550 (2), 331 (25), 289 (10), 229 (8), 202 (14), 169 (62), 121 (100), 109 (46), 91 (16). $[\alpha]_{\mathrm{D}}{ }^{20}=-103$ (c 0.25, $\mathrm{CHCl}_{3}$ ). $\mathrm{For}_{28} \mathrm{H}_{38} \mathrm{O}_{11}$ (550.61): C 61.08, H 6.96; found: C 61.05, H 6.98.

6a/6b: ${ }^{1} \mathrm{H}-\mathrm{NMR}\left(\mathrm{CDCl}_{3}\right)$ : Diastereoisomer A: 0.88 (m, 1H, H-3a), 1.06 (m, 1H, H-5a), 1.19 (m, 1H, H-6a), 1.20 (m, 1H, H-4a), 1.52 (m, 1H, H-5b), 1.58 (m, 1H, H-2), 1.64 (m, 1H, H-3b), 1.71 (m, 1H, H-4b), 1.95 (m, 1H, H-6b), 2.09 (s, 3H), 2.09-2.14 (m, 4H, H-7a, OAc), 2.12 (s, 3H, OAc), 2.14 (s, $3 \mathrm{H}, \mathrm{OAc}$ ), 2.16 (s, 3H, OAc), 3.19 (dd, $J=3.8,13.4 \mathrm{~Hz}, 1 \mathrm{H}, \mathrm{H}-7 \mathrm{~b}), 3.32$ (dt, $J=4.1,9.5,9.5 \mathrm{~Hz}, 1 \mathrm{H}$, 
H-1), 3.78 (s, 3H, H-12), 3.92 (dt, $J=1.3,5.8,6.8 \mathrm{~Hz}, \mathrm{H}-5$ '), 4.10 (dd, $J=5.8,11.2 \mathrm{~Hz}, 1 \mathrm{H}, \mathrm{H}-6$ 'a), 4.22 (dd, $J=6.8,11.2 \mathrm{~Hz}, 1 \mathrm{H}, \mathrm{H}-6$ 'b), 4.60 (d, $\left.J=8.0 \mathrm{~Hz}, 1 \mathrm{H}, \mathrm{H}-1^{\prime}\right), 5.04$ (dd, $J=3.5,10.5 \mathrm{~Hz}, 1 \mathrm{H}, \mathrm{H}-$ 3'), 5.31 (dd, $J=8.0,10.5 \mathrm{~Hz}, 1 \mathrm{H}, \mathrm{H}-2$ '), 5.39 (dd, $J=1.3,3.5 \mathrm{~Hz}, 1 \mathrm{H}, \mathrm{H}-4$ '), 6.82 (m, 2H, H-10), 7.09 (m, 2H, H-9); Diastereoisomer B: 0.88 (m, 1H, H-3a), 1.06 (m, 1H, H-5a), 1.19 (m, 1H, H-6a), 1.20 (m, 1H, H-4a), 1.52 (m, 1H, H-5b), 1.58 (m, 1H, H-2), 1.64 (m, 1H, H-3b), 1.71 (m, 1H, H-4b), 1.95 (m, 1H, H-6b), 2.03 (s, 3H, OAc), 2.03-2.06 (m, 1H, H-7a), 2.04 (s, 3H, OAc), 2.05 (s, 3H, OAc), 2.06 (s, 3H, OAc), 3.08 (dd, $J=3.3,13.2 \mathrm{~Hz}, 1 \mathrm{H}, \mathrm{H}-7 \mathrm{~b}), 3.22$ (dt, $J=4.3,9.9,9.9 \mathrm{~Hz}, 1 \mathrm{H}, \mathrm{H}-1), 3.78$ (s, $3 \mathrm{H}$, H-12), 3.89 (dt, $J=1.3,6.3,6.3 \mathrm{~Hz}, 1 \mathrm{H}, \mathrm{H}-5$ '), 4.11 (dd, $J=6.2,11.1 \mathrm{~Hz}, 1 \mathrm{H}, \mathrm{H}-6$ 'a), 4.19 (dd, $J=6.3$, $11.1 \mathrm{~Hz}, 1 \mathrm{H}, \mathrm{H}-6$ 'b), 4.56 (d, $J=7.9 \mathrm{~Hz}, 1 \mathrm{H}, \mathrm{H}-1^{\prime}$ ), 5.04 (dd, $J=3.5,10.5 \mathrm{~Hz}, 1 \mathrm{H}, \mathrm{H}-3$ '), 5.20 (dd, $J=$ 7.9, 10.5Hz, 1H, H-2'), 5.39 (dd, $J=1.3,3.5 \mathrm{~Hz}, 1 \mathrm{H}, \mathrm{H}-4$ '), 6.80 (m, 2H, H-10), 7.03 (m, 2H, H-9). IR $\left(\mathrm{cm}^{-1}, \mathrm{CCl}_{4}\right): 3063(\mathrm{w}), 3031(\mathrm{w}), 2935(\mathrm{~m}), 2859(\mathrm{~m}), 2836(\mathrm{w}), 1757(\mathrm{~s}), 1613(\mathrm{w}), 1513(\mathrm{~s}), 1441$ (w), 1369 (s), 1300 (w), 1245 (s), 1221 (s), 1172 (s), 1044 (s), 880 (w), 856 (w). MS (FAB): [M] 550 (1), 331 (16), 202 (18), $169(36), 121$ (100), 109 (34), $81(10) .[\alpha]_{\mathrm{D}}{ }^{20}=-72$ (c 0.21, $\left.\mathrm{CHCl}_{3}\right)$. For $\mathrm{C}_{28} \mathrm{H}_{38} \mathrm{O}_{11}$ (550.61): C 61.08, H 6.96; found: C 60.99, H 6.99.

2-(4-Methoxybenzyl)cyclohexyl- $\beta$-D-glucopyranoside (7a/7b and $\mathbf{9 a} / \mathbf{9 b})$ and 2-(4-methoxybenzyl)cyclohexyl- $\beta$-D-galactopyranoside $(\mathbf{8 a} / \mathbf{8 b}$ and $\mathbf{1 0 a} / \mathbf{1 0 b})$.

A solution of the respective compounds $3 \mathbf{a} / \mathbf{3 b}-\mathbf{6 a} / \mathbf{6 b}(0.206 \mathrm{~g} ; 0.374 \mathrm{mmol})$ in a mixture of methanol $(20 \mathrm{~mL})$ and water $(4 \mathrm{~mL})$ was heated to reflux in the presence of potassium carbonate $(0.3$ $\mathrm{g}$ ) for $2 \mathrm{~h}$. Methanol and water were removed under reduced pressure, and the residue was applied on the top of a column filled with silica gel and purified by elution with a 15:1 to 5:1 stepwise gradient of chloroform / methanol mixture, affording the pure products in yields of $92.4 \%(7 \mathbf{a} / 7 \mathbf{b}), 88.6 \%$ $(\mathbf{8 a} / \mathbf{8 b}), 53.8 \%(\mathbf{9 a} / \mathbf{9 b})$ and $66.4 \%(\mathbf{1 0 a} / \mathbf{1 0 b})$, respectively.

7a/7b: ${ }^{1} \mathrm{H}-\mathrm{NMR}\left(\mathrm{CD}_{3} \mathrm{OD}\right)$ : Diastereoisomer A: 1.24 (m, 1H, H-4a), 1.32 (m, 1H, H-3a), 1.41 (m, 2H, H-5a, H-6a), 1.52 (m, 1H, H-3b), 1.65 (m, 1H, H-4b), 1.72 (m, 1H, H-5b), 1.76 (m, 1H, H-2), 2.04 (m, $1 \mathrm{H}, \mathrm{H}-6 \mathrm{~b}), 2.52$ (dd, $J=8.4,13.7 \mathrm{~Hz}, 1 \mathrm{H}, \mathrm{H}-7 \mathrm{a}), 2.84$ (dd, $J=6.6,13.7 \mathrm{~Hz}, 1 \mathrm{H}, \mathrm{H}-7 \mathrm{~b}), 3.25$ (ddd, $J=$ 2.5, 5.6, 9.7Hz, 1H, H-5'), 3.27 (dd, $J=7.7,9.2 \mathrm{~Hz}, 1 \mathrm{H}, \mathrm{H}-2^{\prime}$ ), 3.32 (t, $J=9.5 \mathrm{~Hz}, 1 \mathrm{H}, \mathrm{H}-4^{\prime}$ ), 3.37 (t, $J$ $\left.=9.2 \mathrm{~Hz}, 1 \mathrm{H}, \mathrm{H}-3^{\prime}\right), 3.72(\mathrm{dd}, J=5.6,11.7 \mathrm{~Hz}, 1 \mathrm{H}, \mathrm{H}-6$ 'a), 3.75 (s, 3H, H-12), 3.89 (dd, $J=2.5$, $11.7 \mathrm{~Hz}, 1 \mathrm{H}, \mathrm{H}-6$ 'b), 3.92 (dt, $J=2.5,2.5,4.9 \mathrm{~Hz}, 1 \mathrm{H}, \mathrm{H}-1), 4.34$ (d, $J=7.7 \mathrm{~Hz}, 1 \mathrm{H}, \mathrm{H}-1$ '), 6.79 (m, 2H, H-10), 7.15 (m, 2H, H-9); Diastereoisomer B: 1.24 (m, 1H, H-4a), 1.32 (m, 2H, H-3a, H-6a), 1.36 (m, 1H, H-5a), 1.52 (m, 1H, H-3b), 1.65 (m, 1H, H-4b), 1.70 (m, 1H, H-2), 1.77 (m, 1H, H-5b), 1.96 (m, $1 \mathrm{H}, \mathrm{H}-6 \mathrm{~b}), 2.42$ (dd, $J=7.9,13.6 \mathrm{~Hz}, 1 \mathrm{H}, \mathrm{H}-7 \mathrm{a}), 2.81$ (dd, $J=6.5,13.6 \mathrm{~Hz}, 1 \mathrm{H}, \mathrm{H}-7 \mathrm{~b}), 3.20$ (ddd, $J=$ 2.4, 5.7, 9.6Hz, 1H, H-5'), 3.25 (dd, $J=7.8,9.1 \mathrm{~Hz}, 1 \mathrm{H}, \mathrm{H}-2^{\prime}$ ), 3.27 (t, $J=9.7 \mathrm{~Hz}, 1 \mathrm{H}, \mathrm{H}-4$ '), 3.35 (dd, $J=9.2,9.8 \mathrm{~Hz}, 1 \mathrm{H}, \mathrm{H}-3$ ') 3.65 (dd, $J=5.7,11.8 \mathrm{~Hz}, 1 \mathrm{H}, \mathrm{H}-6$ 'a), 3.73 (dt, $J=2.4,2.4,4.8 \mathrm{~Hz}, 1 \mathrm{H}, \mathrm{H}-$ 1), 3.75 (s, 3H, H-12), 3.82 (dd, $J=2.4,11.8 \mathrm{~Hz}, 1 \mathrm{H}, \mathrm{H}-6$ 'b), 4.33 (d, $J=7.8 \mathrm{~Hz}, 1 \mathrm{H}, \mathrm{H}-1$ '), 6.80 (m, 2H, H-10), 7.15 (m, 2H, H-9). IR (cm $\left.{ }^{-1}, \mathrm{KBr}\right): 3401$ (s), 2994 (w), 2932 (s), 1612 (m), 1513 (s), 1177 (s), 1096 (s), 1074 (s), 1036 (s), 1018 (s), 895 (w), 842 (w). MS (FAB): [M+H] 383 (1), 325 (1), 273 (1), 261 (1), 241 (1), 203 (16), 121 (100), 91 (5), 79 (7). [ $\alpha]_{\mathrm{D}}^{20}=-63$ (c 0.19, $\left.\mathrm{CH}_{3} \mathrm{OH}\right)$. For $\mathrm{C}_{20} \mathrm{H}_{30} \mathrm{O}_{7}$ (382.46): C 62.81, H 7.91; found: C 62.75, H 7.92. 
8a/8b: ${ }^{1} \mathrm{H}-\mathrm{NMR}\left(\mathrm{CD}_{3} \mathrm{OD}\right)$ : Diastereoisomer A: 1.25 (m, 1H, H-4a), 1.33 (m, 1H, 3a), 1.37 (m, 1H, H5a), 1.41 (m, 1H, H-6a), 1.52 (m, 1H, H-3b), 1.66 (m, 1H, H-4b), 1.72 (m, 1H, H-5b), 1.76 (m, 1H, H2), $2.02(\mathrm{~m}, 1 \mathrm{H}, \mathrm{H}-6 \mathrm{~b}), 2.52$ (dd, $J=8.4,13.6 \mathrm{~Hz}, 1 \mathrm{H}, \mathrm{H}-7 \mathrm{a}), 2.85$ (dd, $J=6.6,13.6 \mathrm{~Hz}, 1 \mathrm{H}, \mathrm{H}-7 \mathrm{~b})$, 3.48 (dd, $J=3.5,9.7 \mathrm{~Hz}, 1 \mathrm{H}, \mathrm{H}-3$ ') 3.48 (dt, $J=1.2,6.2,6.2 \mathrm{~Hz}, 1 \mathrm{H}, \mathrm{H}-5$ '), 3.60 (dd, $J=7.7,9.7 \mathrm{~Hz}$, $1 \mathrm{H}, \mathrm{H}-2$ ') 3.75 (s, 3H, H-12), 3.76 (dd, $J=6.1,11.0 \mathrm{~Hz}, 1 \mathrm{H}, \mathrm{H}-6$ 'a), 3.80 (dd, $J=6.3,11.0 \mathrm{~Hz}, 1 \mathrm{H}, \mathrm{H}-$ 6'b), 3.88 (dt, $J=1.2,3.5 \mathrm{~Hz}, 1 \mathrm{H}, \mathrm{H}-4$ ') 3.91 (dt, $J=2.4,2.4,4.9 \mathrm{~Hz}, 1 \mathrm{H}, \mathrm{H}-1$ ), 4.30 (d, $J=7.7 \mathrm{~Hz}, 1 \mathrm{H}$, H-1'), 6.79 (m, 2H, H-10), 7.15 (m, 2H, H-9); Diastereoisomer B: 1.25 (m, 1H, H-4a), 1.31 (m, 1H, H6a), 1.33 (m, 1H, H-3a), 1.35 (m, 1H, H-5a), 1.52 (m, 1H, H-3b), 1.66 (m, 1H, H-4b), 1.69 (m, 1H, H2), 1.78 (m, 1H, H-5b), 1.96 (m, 1H, H-6b), 2.42 (dd, $J=7.8,13.7 \mathrm{~Hz}, 1 \mathrm{H}, \mathrm{H}-7 \mathrm{a}), 2.81$ (dd, $J=6.6$, 13.7Hz, 1H, H-7b), 3.44 (dt, $J=1.2,6.2,6.2 \mathrm{~Hz}, 1 \mathrm{H}, \mathrm{H}-5$ '), 3.48 (dd, $J=3.4,9.7 \mathrm{~Hz}, 1 \mathrm{H}, \mathrm{H}-3$ '), 3.58 (dd, $J=7.8,9.7 \mathrm{~Hz}, 1 \mathrm{H}, \mathrm{H}-2$ ') 3.69 (dd, $J=6.4,11.1 \mathrm{~Hz}, 1 \mathrm{H}, \mathrm{H}-6$ 'a), 3.72 (dd, $J=6.1,11.1 \mathrm{~Hz}, 1 \mathrm{H}, \mathrm{H}-$ 6’b), 3.74 (dt, $J=2.4,2.4,4.8 \mathrm{~Hz}, 1 \mathrm{H}, \mathrm{H}-1$ ), 3.75 (s, 3H, H-12), 3.84 (dd, $J=1.1,3.4 \mathrm{~Hz}, 1 \mathrm{H}, \mathrm{H}-4$ '), 4.28 (d, $J=7.8 \mathrm{~Hz}, 1 \mathrm{H}, \mathrm{H}-1$ '), 6.80 (m, 2H, H-10), 7.14 (m, 2H, H-9). IR (cm $\left.{ }^{-1}, \mathrm{KBr}\right): 3413$ (s), 2923 (s), 2908 (m), 1611 (w), 1512 (s) 1105 (m), 1090 (s), 1082 (s), 1049 (s), 1038 (s), 848 (w). MS (FAB): $[\mathrm{M}+\mathrm{H}]^{+} 383(1), 231(2), 203(9), 121(100), 91(8), 79(17) .[\alpha]_{\mathrm{D}}^{20}=-82\left(c 0.20, \mathrm{CH}_{3} \mathrm{OH}\right)$. For $\mathrm{C}_{20} \mathrm{H}_{30} \mathrm{O}_{7}$ (382.46): C 62.81, H 7.91; found: C 62.87, H 7.85.

9a/9b: ${ }^{1} \mathrm{H}-\mathrm{NMR}\left(\mathrm{CD}_{3} \mathrm{OD}\right)$ : Diastereoisomer A: 0.90 (m, 1H, H-3a), 1.08 (m, 1H, H-5a), 1.22 (m, 1H, H-6a), 1.24 (m, 1H, H-4a), 1.55 (m, 1H, H-5b), 1.58 (m, 1H, H-2), 1.60 (m, 1H, H-3b), 1.73 (m, 1H, H-4b), 2.13 (m, 1H, H-6b), 2.22 (dd, $J=9.4,13.7 \mathrm{~Hz}, 1 \mathrm{H}, \mathrm{H}-7 \mathrm{a}), 3.23-3.41$ (m, 3H, H-3', 4' and 5'), 3.24 (dd, $J=7.8,9.2 \mathrm{~Hz}, 1 \mathrm{H}, \mathrm{H}-2$ ') 3.39 (dd, $J=2.9,13.7 \mathrm{~Hz}, 1 \mathrm{H}, \mathrm{H}-7 \mathrm{~b}), 3.49$ (dt, $J=4.1,9.6,9.6 \mathrm{~Hz}$, $1 \mathrm{H}, \mathrm{H}-1), 3.70$ (dd, $J=5.6,11.7 \mathrm{~Hz}, 1 \mathrm{H}, \mathrm{H}-6$ 'a), 3.75 (s, 3H, H-12), 3.90 (dd, $J=2.3,11.7 \mathrm{~Hz}, 1 \mathrm{H}, \mathrm{H}-$ 6’b), 4.41 (d, J = 7.8Hz, 1H, H-1'), 6.80 (m, 2H, H-10), 7.09 (m, 2H, H-9); Diastereoisomer B: 0.90 (m, 1H, H-3a), 1.08 (m, 1H, H-5a), 1.22 (m, 1H, H-6a), 1.24 (m, 1H, H-4a), 1.55 (m, 1H, H-5b), 1.58 (m, 1H, H-2), 1.60 (m, 1H, H-3b), 1.73 (m, 1H, H-4b), 2.13 (m, 1H, H-6b), 2.15 (dd, J = 10.0, 13.3Hz, 1H, H-7a), 3.22 (dd, $J=7.8,9.2,1 \mathrm{H}, \mathrm{H}-2^{\prime}$ '), 3.23 - 3.41 (m, 4H, H-7b, H-3', H-4' and H-5'), 3.68 (dd, $J=5.2,11.6 \mathrm{~Hz}, 1 \mathrm{H}, \mathrm{H}-6$ 'a), 3.75 (s, 3H, H-12), 3.85 (dd, J = 2.2, 11.7Hz, 1H, H-6'b), 4.40 (d, $J=$ 7.8Hz, 1H, H-1'), 6.80 (m, 2H, H-10), 7.08 (m, 2H, H-9). IR (cm $\left.{ }^{-1}, \mathrm{KBr}\right): 3429$ (s), 2995 (w), 2927 (m), 1612 (m), 1513 (m), 1099 (m), 1074 (m), 1036 (s), 1024 (s), 879 (w). MS (FAB): [M+H] 383 (1), $261(1), 241(2), 203(8), 121(100), 91(5), 79(16)$. [ $\alpha]_{\mathrm{D}}{ }^{20}=-67$ (c 0.26, $\left.\mathrm{CH}_{3} \mathrm{OH}\right)$. For $\mathrm{C}_{20} \mathrm{H}_{30} \mathrm{O}_{7}$ (382.46): C 62.81, H 7.91; found: C 62.85, H 7.88.

10a/10b: ${ }^{1} \mathrm{H}-\mathrm{NMR}\left(\mathrm{CD}_{3} \mathrm{OD}\right)$ : Diastereoisomer A: 0.89 (m, 1H, H-3a), 1.08 (m, 1H, H-5a), 1.23 (m, 1H, H-4a), 1.37 (m, 1H, H-6a), 1.56 (m, 1H, 5b), 1.61 (m, 2H, H-2, H-3b), 1.72 (m, 1H, 4b), 2.14 (dd, $J=9.9,13.4 \mathrm{~Hz}, 1 \mathrm{H}, \mathrm{H}-7 \mathrm{a}), 2.22$ (m, 1H, H-6b), 3.40 (dd, $J=3.3,13.4 \mathrm{~Hz}, 1 \mathrm{H}), 3.48(\mathrm{~m}, 1 \mathrm{H}), 3.48$ (dd, $J=3.3,9.7 \mathrm{~Hz}, 1 \mathrm{H}), 3.50(\mathrm{dt}, J=1.1,6.2,6.2 \mathrm{~Hz}, 1 \mathrm{H}), 3.55(\mathrm{dd}, J=7.5,9.7 \mathrm{~Hz}, 1 \mathrm{H}), 3.75(\mathrm{~s}, 3 \mathrm{H}), 3.75$ $(\mathrm{dd}, J=6.2,11.2 \mathrm{~Hz}, 1 \mathrm{H}), 3.78(\mathrm{dd}, J=6.3,11.2 \mathrm{~Hz}, 1 \mathrm{H}), 3.86(\mathrm{dd}, J=1.1,3.3 \mathrm{~Hz}, 1 \mathrm{H}), 4.36(\mathrm{~d}, J=$ 7.5Hz, 1H), 6.79 (m, 2H), 7.09 (m, 2H); Diastereoisomer B: 0.89 (m, 1H, H-3a), 1.08 (m, 1H, H-5a), 1.23 (m, 1H, H-4a), 1.28 (m, 1H, H-6a), 1.56 (m, 1H, 5b), 1.57 (m, 1H, H-2), 1.61 (m, 1H, H-3b), 1.72 (m, 1H, 4b), 2.14 (m, 1H, H-6b), 2.21 (dd, $J=9.5,13.7 \mathrm{~Hz}, 1 \mathrm{H}, \mathrm{H}-7 \mathrm{a}), 3.24$ (dd, $J=3.6,13.7 \mathrm{~Hz}, 1 \mathrm{H}$, H-7b), 3.32 (m, 1H, H-1), 3.47 (dd, $J=3.4,9.7 \mathrm{~Hz}, 1 \mathrm{H}, \mathrm{H}-3$ '), 3.50 (dt, $J=1.0,6.2,6.2 \mathrm{~Hz}, 1 \mathrm{H}, \mathrm{H}-5^{\prime}$ ), 3.57 (dd, $J=7.7,9.7 \mathrm{~Hz}, 1 \mathrm{H}, \mathrm{H}-2$ '), 3.72 (dd, $J=6.1,11.2 \mathrm{~Hz}, 1 \mathrm{H}, \mathrm{H}-6$ 'a), 3.75 (s, 3H, H-12), 3.75 (dd, 
$J=6.3,11.2 \mathrm{~Hz}, 1 \mathrm{H}, \mathrm{H}-6$ 'b), 3.85 (dd, $J=1.0,3.4 \mathrm{~Hz}, 1 \mathrm{H}, \mathrm{H}-4$ '), 4.36 (d, $J=7.7 \mathrm{~Hz}, 1 \mathrm{H}, \mathrm{H}-1$ '), 6.80 (m, 2H, H-10), 7.08 (m, 2H, H-9). IR (cm $\left.{ }^{-1}, \mathrm{KBr}\right): 3520$ (s), 3420 (s), 2990 (w), 1613 (m), 1514 (s) 1100 (m), 1084 (s), 1078 (m), 1052 (s), 1035 (s), 880 (w). MS (FAB): [M+H] 383 (1), 231 (1), 203 (10), 121 (100), $91(8), 79(11) .[\alpha]_{\mathrm{D}}{ }^{20}=-86\left(c 0.25, \mathrm{CH}_{3} \mathrm{OH}\right)$. For $\mathrm{C}_{20} \mathrm{H}_{30} \mathrm{O}_{7}$ (382.46): $\mathrm{C}$ 62.81, $\mathrm{H}$ 7.91; found: C $62.78, \mathrm{H} 7.87$.

Table 1. ${ }^{13} \mathrm{C}-\mathrm{NMR}$ data of the protected alkyl glycosides $\mathbf{3 a}-\mathbf{6 b}$ (measured in $\mathrm{CDCl}_{3}$ )

\begin{tabular}{|c|c|c|c|c|c|c|c|c|}
\hline \multirow{2}{*}{$\begin{array}{c}\text { Carbon } \\
\text { atom } \\
\text { No. }\end{array}$} & \multicolumn{8}{|c|}{ Compound } \\
\hline & $3 \mathbf{a}^{\mathrm{a}}$ & $3 b^{a}$ & $4 a^{b}$ & $4 b^{b}$ & $5 \mathbf{a}^{\mathrm{c}}$ & $5 b^{c}$ & $6 \mathbf{a}^{\mathrm{d}}$ & $6 \mathbf{b}^{\mathrm{d}}$ \\
\hline 1 & $74.65(\mathrm{~d})$ & 79.93 (d) & 75.18 (d) & 80.07 (d) & 80.94 (d) & $85.60(\mathrm{~d})$ & 81.25 (d) & 85.77 (d) \\
\hline 2 & $43.89(\mathrm{~d})$ & $43.80(\mathrm{~d})$ & $43.81(\mathrm{~d})$ & $43.72(d)$ & $45.14(d)$ & $44.32(\mathrm{~d})$ & $45.12(\mathrm{~d})$ & $44.28(\mathrm{~d})$ \\
\hline 3 & $26.82(t)$ & $26.68(t)$ & $26.73(t)$ & $26.62(\mathrm{t})$ & $29.76(t)$ & $29.68(t)$ & $29.76(t)$ & $29.60(\mathrm{t})$ \\
\hline 4 & $25.37(\mathrm{t})$ & $25.06(\mathrm{t})$ & $25.30(\mathrm{t})$ & $24.96(\mathrm{t})$ & $24.76(\mathrm{t})$ & $24.43(\mathrm{t})$ & $24.76(\mathrm{t})$ & $24.41(\mathrm{t})$ \\
\hline 5 & $20.76(t)$ & $20.70(\mathrm{t})$ & $20.98(\mathrm{t})$ & $20.84(\mathrm{t})$ & $24.91(\mathrm{t})$ & $24.88(\mathrm{t})$ & $24.93(\mathrm{t})$ & $24.81(\mathrm{t})$ \\
\hline 6 & $31.93(\mathrm{t})$ & $28.78(\mathrm{t})$ & $31.93(\mathrm{t})$ & $28.88(\mathrm{t})$ & $33.72(\mathrm{t})$ & $31.23(\mathrm{t})$ & $33.79(\mathrm{t})$ & $31.26(\mathrm{t})$ \\
\hline 7 & $37.23(\mathrm{t})$ & $37.17(\mathrm{t})$ & $37.22(\mathrm{t})$ & $37.03(\mathrm{t})$ & $37.30(\mathrm{t})$ & $37.26(t)$ & $37.34(\mathrm{t})$ & $37.31(\mathrm{t})$ \\
\hline 8 & $133.43(\mathrm{~s})$ & $133.01(\mathrm{~s})$ & $133.51(\mathrm{~s})$ & $133.04(\mathrm{~s})$ & $132.91(\mathrm{~s})$ & $132.44(\mathrm{~s})$ & $132.99(\mathrm{~s})$ & $132.47(\mathrm{~s})$ \\
\hline 9 & $130.30(d)$ & $129.84(\mathrm{~d})$ & $130.27(d)$ & $129.84(d)$ & $130.40(d)$ & $130.08(d)$ & $130.35(\mathrm{~d})$ & $130.10(\mathrm{~d})$ \\
\hline 10 & $113.78(d)$ & $113.34(\mathrm{~d})$ & $113.77(\mathrm{~d})$ & $113.40(\mathrm{~d})$ & $113.64(\mathrm{~d})$ & $113.41(\mathrm{~d})$ & $113.64(\mathrm{~d})$ & $113.44(\mathrm{~d})$ \\
\hline 11 & $157.83(\mathrm{~s})$ & $157.67(\mathrm{~s})$ & $157.83(\mathrm{~s})$ & $157.65(\mathrm{~s})$ & $157.81(\mathrm{~s})$ & $157.66(\mathrm{~s})$ & $157.80(\mathrm{~s})$ & $157.66(\mathrm{~s})$ \\
\hline 12 & $55.24(q)$ & $55.19(q)$ & $55.24(q)$ & $55.21(q)$ & $55.21(q)$ & $55.21(q)$ & $55.21(q)$ & $55.21(\mathrm{q})$ \\
\hline 1 ' & $101.76(d)$ & $98.13(d)$ & $102.32(d)$ & $98.85(\mathrm{~d})$ & $102.04(d)$ & $98.49(\mathrm{~d})$ & $102.56(d)$ & $99.24(\mathrm{~d})$ \\
\hline 2 ' & $71.76(\mathrm{~d})$ & 7159 (d) & $69.34(d)$ & $69.19(\mathrm{~d})$ & 71.67 (d) & 7158 (d) & $69.32(d)$ & $69.25(\mathrm{~d})$ \\
\hline $3^{\prime}$ & $73.11(\mathrm{~d})$ & $73.08(d)$ & 71.17 (d) & $71.16(\mathrm{~d})$ & 73.09 (d) & $73.05(\mathrm{~d})$ & 71.18 (d) & 71.18 (d) \\
\hline $4^{\prime}$ & $68.94(\mathrm{~d})$ & $68.67(d)$ & $67.21(\mathrm{~d})$ & $67.19(\mathrm{~d})$ & 68.77 (d) & $68.75(\mathrm{~d})$ & $67.13(d)$ & $67.13(d)$ \\
\hline 5 & 71.59 (d) & $71.52(d)$ & 70.48 (d) & $70.45(\mathrm{~d})$ & 71.79 (d) & $71.60(\mathrm{~d})$ & $70.52(d)$ & $70.46(d)$ \\
\hline 6 ' & $62.28(\mathrm{t})$ & $62.10(\mathrm{t})$ & $61.37(\mathrm{t})$ & $61.36(\mathrm{t})$ & $62.27(\mathrm{t})$ & $62.10(\mathrm{t})$ & $61.36(\mathrm{t})$ & $61.36(\mathrm{t})$ \\
\hline
\end{tabular}


Signals of the protecting acetate functionalities on the carbohydrate part of the molecules: ${ }^{\mathrm{a}} 20.60$ (q), 20.60 (q), 20.64 (q), 20.64 (q), 20.70 (q), 20.70 (q), 20.76 (q), 20.84 (q), 169.20 (s), 169.23 (s), 169.43 (s), 169.45 (s), 170.37 (s), 170.40 (s), 170.59 (s), 170.76 (s); ${ }^{b} 20.61$ (q), 20.62 (q), 20.62 (q), 20.66 (q), 20.71 (q), 20.71 (q), 20.73 (q), 20.90 (q), 169.95 (s), 170.21 (s), 170.26 (s), 170.39 (s), 170.39 (s), 170.40 (s), 170.43 (s), 170.48 (s); 20.60 (q), 20.60 (q), 20.60 (q), 20.63 (q), 20.69 (q), 20.69 (q), 20.69 (q), 20.71 (q), 169.27 (s), 169.32 (s), 169.41 (s), 169.42 (s), 170.35 (s), 170.37 (s), 170.62 (s), 170.68 (s); ${ }^{\mathrm{d}} 20.60$ (q), 20.62 (q), 20.64 (q), 20.69 (q), 20.70 (q), 20.70 (q), 20.80 (q), 20.83 (q), 169.40 (s), 169.86 (s), 169.95 (s), 170.22 (s), 170.35 (s), 170.38 (s), 170.40 (s), 170.48 (s).

Table 2. ${ }^{13} \mathrm{C}-\mathrm{NMR}$ data of the alkyl glycosides $\mathbf{7 a}-\mathbf{1 0 b}$ (measured in $\mathrm{CD}_{3} \mathrm{OD}$ )

\begin{tabular}{|c|c|c|c|c|c|c|c|c|}
\hline \multirow{2}{*}{$\begin{array}{c}\text { Carbon } \\
\text { atom } \\
\text { No. } \\
\end{array}$} & \multicolumn{8}{|c|}{ Compound } \\
\hline & $7 \mathbf{a}$ & $7 b$ & $8 \mathbf{a}$ & $8 \mathbf{b}$ & 9a & $9 \mathrm{~b}$ & $10 a$ & $10 \mathrm{~b}$ \\
\hline 1 & $76.58(d)$ & $80.54(\mathrm{~d})$ & 76.48 (d) & $80.55(\mathrm{~d})$ & $81.22(\mathrm{~d})$ & $85.90(\mathrm{~d})$ & $81.26(\mathrm{~d})$ & $85.84(\mathrm{~d})$ \\
\hline 2 & $45.61(d)$ & $45.24(\mathrm{~d})$ & $45.71(\mathrm{~d})$ & $45.33(d)$ & $47.44(\mathrm{~d})$ & $46.72(d)$ & $47.46(\mathrm{~d})$ & $46.73(\mathrm{~d})$ \\
\hline 3 & $28.30(t)$ & $28.23(\mathrm{t})$ & $28.30(\mathrm{t})$ & $28.27(\mathrm{t})$ & $31.57(\mathrm{t})$ & $31.38(\mathrm{t})$ & $31.58(\mathrm{t})$ & $31.39(\mathrm{t})$ \\
\hline 4 & $26.59(\mathrm{t})$ & $26.09(\mathrm{t})$ & $26.55(\mathrm{t})$ & $26.14(t)$ & $26.43(t)$ & $26.21(t)$ & $26.43(t)$ & $26.22(t)$ \\
\hline 5 & $23.30(\mathrm{t})$ & $22.34(\mathrm{t})$ & $23.28(\mathrm{t})$ & $22.37(\mathrm{t})$ & $26.85(t)$ & $26.70(t)$ & $26.86(t)$ & $26.71(t)$ \\
\hline 6 & $33.25(\mathrm{t})$ & $30.13(\mathrm{t})$ & $33.29(\mathrm{t})$ & $30.18(\mathrm{t})$ & $35.71(t)$ & $32.73(\mathrm{t})$ & $35.75(t)$ & $32.78(\mathrm{t})$ \\
\hline 7 & $38.36(\mathrm{t})$ & $37.41(\mathrm{t})$ & $38.25(\mathrm{t})$ & $37.46(t)$ & $39.03(\mathrm{t})$ & $38.99(t)$ & $39.01(t)$ & $39.01(t)$ \\
\hline 8 & $135.61(\mathrm{~s})$ & $135.44(\mathrm{~s})$ & $135.65(\mathrm{~s})$ & $135.49(\mathrm{~s})$ & $135.14(\mathrm{~s})$ & $134.95(\mathrm{~s})$ & $135.13(\mathrm{~s})$ & $134.99(\mathrm{~s})$ \\
\hline 9 & 131.79 (d) & $131.69(\mathrm{~d})$ & $131.81(\mathrm{~d})$ & $131.70(d)$ & $131.84(\mathrm{~d})$ & $131.76(d)$ & $131.86(\mathrm{~d})$ & $131.76(d)$ \\
\hline 10 & $115.04(\mathrm{~d})$ & $114.95(\mathrm{~d})$ & $115.03(d)$ & 114.93 (d) & 114.99 (d) & $114.95(\mathrm{~d})$ & $114.98(\mathrm{~d})$ & $114.93(d)$ \\
\hline 11 & $159.70(\mathrm{~s})$ & $159.64(\mathrm{~s})$ & $159.68(\mathrm{~s})$ & $159.62(\mathrm{~s})$ & $159.70(\mathrm{~s})$ & $159.66(\mathrm{~s})$ & $159.69(\mathrm{~s})$ & $159.65(\mathrm{~s})$ \\
\hline 12 & $56.12(q)$ & $56.12(q)$ & $56.12(q)$ & $56.12(q)$ & $56.12(q)$ & $56.12(q)$ & $56.12(q)$ & $56.12(q)$ \\
\hline 1 , & $105.66(\mathrm{~d})$ & $102.23(\mathrm{~d})$ & $106.32(\mathrm{~d})$ & $102.98(\mathrm{~d})$ & $106.20(d)$ & $101.81(\mathrm{~d})$ & $106.83(d)$ & $102.54(d)$ \\
\hline 2 & 76.08 (d) & $75.77(\mathrm{~d})$ & $73.54(d)$ & $73.24(\mathrm{~d})$ & $76.13(\mathrm{~d})$ & $75.68(\mathrm{~d})$ & $73.57(\mathrm{~d})$ & $73.13(d)$ \\
\hline $3^{\prime}$ & 78.83 (d) & 78.83 (d) & $75.74(\mathrm{~d})$ & $75.70(\mathrm{~d})$ & $78.76(\mathrm{~d})$ & 78.74 (d) & $75.66(\mathrm{~d})$ & $75.64(d)$ \\
\hline 4 & 72.39 (d) & 72.29 (d) & $70.75(\mathrm{~d})$ & $70.75(\mathrm{~d})$ & $72.42(\mathrm{~d})$ & $72.21(\mathrm{~d})$ & $70.81(\mathrm{~d})$ & $70.75(\mathrm{~d})$ \\
\hline 5 , & 78.29 (d) & 78.22 (d) & $76.90(d)$ & $76.86(d)$ & 78.41 (d) & 78.28 (d) & 77.01 (d) & $76.92(d)$ \\
\hline $6^{\prime}$ & $63.47(\mathrm{t})$ & $63.35(\mathrm{t})$ & $62.83(\mathrm{t})$ & $62.83(\mathrm{t})$ & $63.52(\mathrm{t})$ & $63.31(\mathrm{t})$ & $62.92(\mathrm{t})$ & $62.87(\mathrm{t})$ \\
\hline
\end{tabular}




\section{References}

1. Igarashi, K. Adv. Carbohydr. Chem. Biochem. 1977, 34, 243-283.

2. Schmidt, R. R. Angew. Chem. Int. Ed. Engl. 1986, 25, 212-235.

3. Schmidt, R. R.; Jung, K.-H. In 'Preparative Carbohydrate Chemistry'; Hanessian, S., Ed.; Dekker: New York, 1997; pp. 283-312.

4. Nicolaou, K. C.; Ueno, H. In 'Preparative Carbohydrate Chemistry'; Hanessian, S., Ed.; Dekker: New York, 1997; pp. 313-338.

5. Hanessian, S. In 'Preparative Carbohydrate Chemistry'; Hanessian, S. Ed.; Dekker: New York, 1997; pp. 381-388.

6. Wimmer, Z.; Romaňuk, M. Coll. Czech. Chem. Commun. 1982, 47, 1878-1883.

7. Wimmer, Z.; Rejzek, M.; Zarevúcka, M.; Kuldová, J.; Hrdý, I.; Němec, V.; Romaňuk, M. J. Chem. Ecol. 1997, 23, 605-628.

8. Plattner, J. J.; Gless, R. D.; Rapoport, H. J. Am. Chem. Soc. 1972, 94, 8613-8615.

9. Buděšínský, M.; Šaman, D.; Wimmer Z. Coll. Czech. Chem. Commun. 1982, 47, 2763-2767.

10. von Kienlin, M.; Moonen, C. T. W.; van der Toorn, A.; van Zij1, C. M. J. Magn. Reson. 1991, 93, 423-429.

11. Willker, W.; Leibfritz, D.; Kerssebaum, R.; Bermel, W. Magn. Reson. Chem. 1993, 31, 287-292.

Sample availability: Available from the corresponding author (ZW).

(C) 2004 by MDPI (http://www.mdpi.org). Reproduction is permitted for noncommercial purposes. 